\title{
Greenhouse Climate Controller by Using of Internet of Things Technology and Fuzzy Logic
}

\author{
Abdelhakim Sahour $^{1,2}$, Farouk Boumehrez ${ }^{1,3^{*}}$, Mohamed Benouaret $^{4}$, Azzouz Mokhneche $^{4}$ \\ ${ }^{1}$ Department of industrial Engineering, Faculty of Sciences and Technologies, ABBES Laghrour University, Khenchela 40004, \\ Algeria \\ ${ }^{2}$ Laboratoire Systèmes et Applications des Technologies de L'information et des Télécommunications (SATIT), Department of \\ Industrial Engineering, ABBES Laghrour University, Khenchela 40004, Algeria \\ ${ }^{3}$ Laboratoire des Télécommunications (LT), Faculty of Sciences and Technologies, 8 Mai 1945 University, Guelma 24000, \\ Algeria \\ ${ }^{4}$ Department of Electronics, Faculty of Engineering Sciences, University of Annaba, B.P.12, Annaba 23000, Algeria
}

Corresponding Author E-mail: boumehrez.farouk@univ-khenchela.dz

https://doi.org/10.18280/i2m.200105

Received: 27 July 2020

Accepted: 4 December 2020

\section{Keywords:}

ARDUINO uno, fuzzy logic, internet of things, WIFI, wireless sensor network, Zigbee

\begin{abstract}
The Internet of Things (IoT) is a new, ongoing revolution. It often uses wireless sensor network (WSN) technologies because these technologies are among the most important solutions for monitoring and controlling the systems. This article provides a model of a smart greenhouse. In this regard, the main contribution of this paper is an innovative implementation of a micro-climate controlled environment for optimal plant growth, based on loT technology and using a fuzzy logic controller. Using this system, we attempted to optimize the functionality of the system proposed by exploiting an Arduino UNO board for data acquisition and processing. The input variables are analog values captured by ZigBee wireless network sensors that are then processed using fuzzy logic control software with heating and extractor control signals. At the same time, all data were sent to the server through a Wi-Fi internet connection, which permitted remote monitoring and analysis of the data via a web browser with tablets, smartphones, and laptops. Results show that the choice of a fuzzy logic controller could promote a comfortable greenhouse micro-climate. Also, we showed the efficiency of our proposed solution for greenhouse climate remote monitoring anywhere via IoT technology.
\end{abstract}

\section{INTRODUCTION}

By the year 2050, the world will face a significant challenge related to ensuring the availability of food for 9.8 billion humans $[1,2]$ and will require new agricultural land roughly equivalent to the size of Brazil. Any less agricultural land will cause cultivators great difficulty in producing more food, especially in the developing world [2,3], and that type of environmental change increasingly influences agriculture, posing real challenges to this vital sector.

In this vein, the greenhouse is an important part of modern agriculture that can make agricultural production independent from climate and geographical constraints, greatly improving crop yield and their listing period [4]. Thus, the control and management of greenhouses have become an essential part of agricultural automation.

Traditional greenhouses depend on human interventions to control environmental parameters, as a result, energy is lost and optimal production not reached.

This circumstance is an opportunity for many researchers and innovators who are working to develop and introduce innovative agricultural methods in an attempt to use technology to find solutions to these challenges. One of the potential solutions is raising the level of vertical farming [4], which is a great solution for growing large amounts of food in a limited agricultural space. A special technology can be used on vertical cultivation within the farms to control the various factors, such as the lighting, water, etc. In addition, traditional greenhouse acquisition and control systems require the arrangement of a great number of connecting cables. The high temperatures and humidity of the greenhouse can quickly damage these cables. Therefore, the need is urgent for the development of wireless sensors and reliable wireless control systems. These two latter are easy to use instead of sensors connected by wires [5]

Nowadays, smart agriculture is a must, not for farmer laziness but for optimization production, as $70 \%$ of the farming time is spent supervision and understanding the crop cases instead of doing effective fieldwork. Such development will be possible when the agricultural field exploits new information and communication technologies to address challenges related to increased agricultural production with less pollution, water consumption, and agricultural inputs [6]. For that, "Smart agriculture" (or "smart farming") is a revolution in indoor vertical farming that enables producers to improve the agricultural task chain while modernizing landbased farms [7].

Smart agriculture is made possible through the contribution of the Internet of Things (IoT). In the IoT model, many objects can be connected in one form or another $[7,8]$. To reach their common goal, these objects must be characterized by a unique address and communicate with standard protocols that allow them to interact and collaborate [9-11]. The IoT is a means of connecting physical things to the Internet network, including 
allowing access to a large amount of remote sensor data that makes it possible to control the physical world from a distance [12]. The use of IoT in greenhouse agriculture participates in its development. In this paper, IoT can monitor and analyze useful information from the greenhouse to the owner/farmer in real-time from anywhere at any time from any active internet device. So, the system collects the data through wireless sensors, inside and outside of the greenhouse. Then these data are processed by a fuzzy logic controller (FLC) algorithm implanted in an Arduino UNO board. The control of greenhouse temperature and humidity is realized by using heating and extractor control output signals. Also, at the same time, data are sent to a database server to be stored and archived for the long term. Furthermore, the user interfaces offer a menu to analyze the greenhouse parameters. It can give the collected data to growers by using a laptop or smartphone connected to the internet.

However, the IoT brings unique challenges, such as the connection and configuration of sensors to applications. Also, since it is not as easy as manually connecting sensors to an application or a form of middleware [13], this enormous amount of sensors in distributed environments [14] will lead to the creation of massive amount of heterogeneous data. As a result, the means of acquiring, integrating, storing, processing, and using these data has become an urgent and important problem.

These challenges are quite different from those of traditional data management, and solving them contributes to the emerging model of "big data" $[12,15]$ and cloud-based platforms $[16,17]$. Indeed, the European Commission predicts that, by 2020, the "Internet of PCs" will be replaced by the IoT $[18,19]$. Moreover, the number of the world population will represent more than 7.6 billion versus exceed 50 billion devices connected to the Internet [18-20]. These devices include personal computers, smartphones, and other hand-held and embedded devices. These most devices contain different sensors and actuators that can sense, perform computations, make intelligent decisions, and transmit useful information through the Internet. This technology will become widely available because of advances in many technologies, including multifunction systems in chips, ubiquitous cloud services, and digital communication provided by cognitive radio [18].

On the other hand, the greenhouse environment system is very complicated as it is a large, nonlinear, multivariable system featuring large disturbance, time variances, uncertainty, and imprecision [21-23]. Besides, the wireless sensor networks deployed for event detection and the greenhouse monitoring sensor networks environment model should be able to detect if particular events of interest have occurred or are about to occur. Hence, appropriate techniques are needed that allow for the describing of events in ways that sensor nodes will be able to translate a physical phenomenon [24], which makes it difficult to set up a precise mathematical model. In contrast, the important characteristics of the Fuzzy logic that makes it suitable for describing WSN events.

A fuzzy control system is a recent approach widely used in many domains, such as agriculture, in particular for greenhouses. Fuzzy systems can maintain internal climates in good enough and can provide to understating energy consumption.

The Fuzzy logic is not based on a precise mathematical model of a controlled system, and it does not require the precise description of a controlled object. Also, it is robust and adaptable and easily learned by an operator. It is much closer to natural ways of thinking than crisp logic, making it easy to implement. Further, it can use with IoT in agriculture [25]. As such, it is very suitable for controlling a complex greenhouse system. Almeida [15] discusses the fundamental technologies of big data and analysis the conception of big data volumes. Somov et al. [3] used the IoT deployment in a tomato greenhouse in Russia. The IoT technologies applied in the cited paper comprise WSN, cloud computing, and artificial intelligence. Putra et al. [9] worked on the implementation of a smart garbage system based on IoT using the ultrasonic sensor within the internet network. Some published papers that have reported on fuzzy control employment to manage the indoor greenhouse micro-climate conditions: Bambang et al. [25] developed a monitoring system for micro-climate management in pilot-scale indoor aeroponics. Also, Mulenga et al. [26] indicated that internal air problems can be made worse by climate change, which in turn alter the outside conditions that affect inside conditions. Mirzaee-Ghaleh et al [27] demonstrated that fuzzy controllers provided the best response, with a lower error and better performance versus disturbances by ambient conditions for relative humidity and temperature indoor studied poultry houses. On the other hand, Manonmani et al. [28] monitored only the temperature and humidity values of a greenhouse using an artificial neural network (ANN) model for effective and high-quality pepper farming in a greenhouse environment. Azaza et al. [29] developed a system that comprises a fuzzy logic technique that regulates energy consumption in the greenhouse using temperature and humidity values. Pahuja et al. [30] implemented a rule-based temperature control system using virtual instrumentation technologies together with the WSN. The temperature was tracked and smartly controlled with software built on the Graphical User Interface (GUI) on the host computer.

So, considering related works cited, it can be established that there is a need to have a suitable regulated micro-climate for different types of crops, which includes four major environmental parameters, namely temperature, humidity, amount of carbon dioxide $\left(\mathrm{CO}_{2}\right)$ level, and light intensity.

In this paper, we aim to build a smart efficient system that monitors and controls internal greenhouse temperature, and humidity, based on the IoT using wireless sensor equipment. However, the key aim of the study is not only the creation of a consistent growing environment, but it also automates and makes the whole system smart to save manufacturing costs. The suggested solution focuses on greenhouse internal temperature and humidity measurement and regulation, but it can be applied to other types of properties, e.g. Dioxide in carbon $\left(\mathrm{CO}_{2}\right)$.

The contributions of the present study can be outlined as follows:

A- The proposed model aimed to develop a micro-climate management system inside the greenhouse.

B- Using a web page that permitted anywhere monitoring internal greenhouse conditions.

The rest of this paper is organized as follows: Sec. 2 presents the Greenhouse monitoring systems. Section 3 provides the structure of the proposed system. Sec. 4 describes the evaluation methods and results. Finally, a conclusion is given in Sec. 5. 


\section{DESIGN OF GREEN HOUSE MONITORING SYSTEM}

Production of crops in greenhouses has become a principal way for growers to control most production parameters. It allows them to achieve better agricultural productivity than in open fields by optimizing land use to provide a variety of fresh vegetables and fruits throughout the year and for a longer duration [31-33].

In fact, important control parameters are [34]:

-Temperature is generally the most important variable to control both the point of plant survival life (a few minutes at extreme temperature can destroy a plant) and of its growth.

-The humidity of the air is also an important variable. A period of several hours at $100 \%$ humidity should be avoided, which can block the transpiration of the plant. Also, avoid too low humidity levels which dry out the plant.

-Soil moisture is less critical for durations of a few hours, but it is an important variable for the plant to be able to feed.

-The level of $\mathrm{CO}_{2}$ must be regulated in very airtight greenhouses which rapidly consume the rate of carbon dioxide during photosynthesis. This $\mathrm{CO}_{2}$ rate can be enriched in cases where there is insufficient sunlight.

-The lighting can also be regulated to control the development of the plant: by means of artificial lighting to accelerate its development, by shade curtain to slow it down.

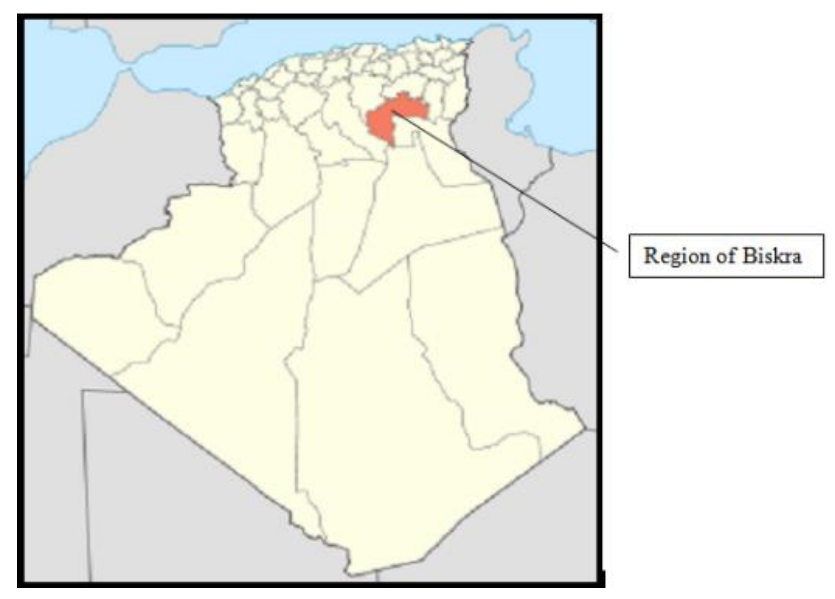

Figure 1. Study area situation

South Algeria is a region of the world known for its extreme climate. Indeed, Biskra is situated in southern Algeria as shown in Figure 1. The city was therefore selected to carry the experiment. Biskra has desert climate which is very hot. The summer is very hot and dry and the winter is mild. [35]. Furthermore, full periods of sunshine normally last a large part of the day. Thus, temperature regulation in the greenhouse is extremely difficult during the portion of the day when the Sun's rays maximize. It is very important to maintain an adequate temperature in a greenhouse setting. In only a few hours, temperature increases will affect the plants, and thus decrease productivity. On the other hand, the Biskra Province is the first producer of early vegetables, which produces $32 \%$ of national protected crop production [35].

In addition, the tunnel greenhouse is the most common structure in this area. In this work, it was used to provide a realistic investigation. There are many arches implanted in the soil and covered with a plastic envelope. The standard dimensions are $8 \mathrm{~m}$ in width, $50 \mathrm{~min}$ length, and $3 \mathrm{~m}$ in height [36].
Generally, only certain variables will be regulated depending on the local climate. Temperature and humidity are considered to among the generality influential parameters in plant production [37]. Indeed, greenhouse temperature has a considerable impact on vegetative growth [38]. The hygrometry desired by a farmer is between $40 \%$ and $60 \%$. Furthermore, humidity varies with temperature; when humidity falls below $40 \%$, the plant transpires more. While it does not destroy the plant, it does quickly deplete its water supply. Also, when the air is too humid, the plant is exposed to attacks from fungus, mold, slowing of transpiration, and slowing of crop growth. Too much moisture is often due to the wrong extraction system or lack of good ventilation in the greenhouse. To improve a greenhouse's profitability, it is essential to optimize the surrounding climate for crops.

The geographical region where the greenhouses are situated is characterized by specific climate conditions. The greenhouse has a special volume. Thus, the ventilation system should provide the entry of carbon dioxide into the greenhouse, and can balance the internal temperature of the greenhouse, which means reducing the high temperatures caused by exaggerated exposure to sunlight. Also, the relative humidity in the greenhouse should be balanced $[35,36]$.

To reach that, it is necessary to control and constantly monitor the climatic parameters mentioned above by installing adequate equipment to heating, ventilation, misting and openings that act preferentially on one factor by modifying the others.

In the present work, we have tried to develop a smart greenhouse control and monitors, based on IoT technologies and embedded multi-chip sensor nodes [39] with fuzzy control, and WSN.

Our proposed solution, allowed the greenhouse to be automatically controlled by measuring parameters with various wireless sensors ingeniously placed in all the space. Collected data on the controller should be processed, then decisions are taken. In this stage, commands are sent to the selected actuator. Via a wireless internet connection, data and real-time decisions are transmitted to the server. Simultaneously, the data are permanently received by the server and saved in a database. This allows the farmer, cultivator, and other users to view the data from a web browser on an internet-connected device (smart laptops, smartphones, etc.). In our proposed design, we chose the most influential greenhouse parameters, namely external (outside) and internal (inside) temperatures, external (outside) and internal (inside) humidity levels, wind speed, and finally, global radiation. However, external experts will be hired to conduct heating and extraction processes to control the two aforementioned variables as clearly shown in Figure 2.

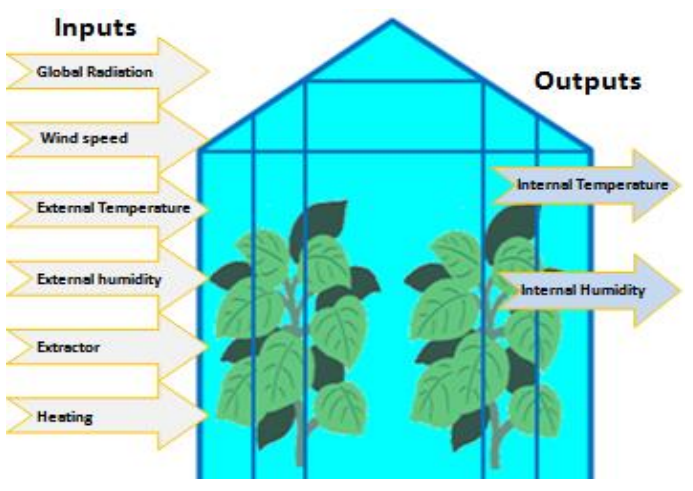

Figure 2. The most influential greenhouse parameters 


\section{THE STRUCTURE OF THE PROPOSED SYSTEM}

The use of modern technologies in protected agriculture assures reduction of the production cost and increases the farmer's profit by increasing productivity in terms of quantity and quality. So, many parameter measurements are required to monitor and control the good quality and productivity of plants in greenhouses. But some very important factors come into play to get the desired results, such as temperature, humidity, light, and water, which are needed for better plant growth [34]

In this paper, the proposed system uses IoT technology in the agricultural area to develop a smart greenhouse, by controlling and monitoring the climatic conditions necessary for plant growth, including internal temperature and humidity. The system employs fuzzy logic implemented in the Arduino UNO board.

The Arduino UNO board collects data (external temperature and internal temperature, external and internal humidity, wind speed, and global radiation) captured by ZigBee wireless network sensors, which are then processed using a fuzzy logic control algorithm with heating and extractor control output signals. The data can also be visualized in the LCD display.

At the same time, all data will be sent to the database server through a Wi-Fi internet connection; these data can be accessed from the cloud with a web browser by authorized users using tablets, smartphones, and laptops. IoT application permittes remote monitoring and analysis of the data.

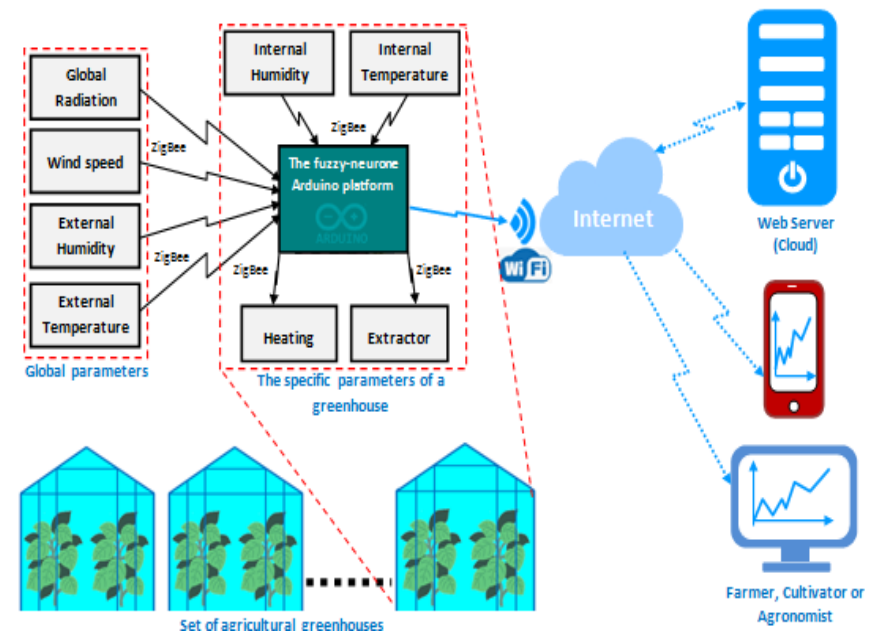

Figure 3. Architectural design of the greenhouse system

The structure of the proposed system is described in Figure 3. The system consists of four parts: (1) wireless sensor network; (2) fuzzy control; (3) processing; and (4) server application parts. The parts are explained in detail in the next section.

\subsection{Wireless sensor network part}

Greenhouses are exigent measurement environments. Previously, most greenhouse monitoring and controls have been wire connected, but the high cost of installation and maintenance has limited the use of that type of system. In a greenhouse, the timeframe of a cultivation cycle is limited. Thus, only wireless sensors designed to operate in severe environments can be used in these environments. Therefore, accuracy, long-term stability, and the integration of the facility are some of the most important factors to consider. Moreover, the shorter lifespan of the cultivation cycle reduces the problem of power consumption, which is the most valuable resource of a WSN.

This type of network directly influences the length of the lifetime of the sensors and, therefore, the entire network.

As indicated above (see Figure 2 and Figure 3), the greenhouse parameters, external and internal temperature, external and internal humidity, wind speed, and global radiation, will be acquired in real-time by deploying six types of sensors. In this study, two sensors were installed inside the greenhouse with specific parameters, and four sensors were installed outside the greenhouse to detect global parameters. Furthermore, the most popular wireless standards are ZigBee, Bluetooth, ultra-wideband (UWB), and Wi-Fi. In refs 31 and 32 a comparative study was considered according to quantitative assessment of the transmission time, data coding efficiency, protocol complexity, power consumption, radio channels, security, etc.

The ZigBee (ZigBee/IEEE 802.15.4 network standard) can provide the design requirements of reliability, security, low cost, low power usage, and capacity, enabling a device to connect to a single network [31-33, 40, 41]. Thus, we chose ZigBee WSN technologies, which are the most suitable for a greenhouse application [42]. The remote sensor nodes are implemented with an XBee module from Digi, which encapsulates the 802.15.4 radio transceiver with the ZigBee protocol stack and the analog sensors. For the global external parameters for all greenhouses, we used the analog resistive temperature and humidity sensors based on product features, such as low power consumption, small size, temperature compensation, calibration microcontroller linear output, low cost, and precise calibration. For example, the DIY Arduino Wind Speed Meter-Anemometer sensor has an analog voltage output that makes it ideal for interfacing with an Arduino; it is rugged and easy to mount.

For global radiation, we used a photocell to sense light. Moreover, each of the sensors was connected to the analog input of an Xbee transceiver. For the specific internal parameters of one greenhouse, we also used resistive temperature and an AMT1001 humidity sensor, connected to the analog input of another Xbee transceiver. Each module is configured as an 'END DEVICE'.

\subsection{Fuzzy control part}

Greenhouses offer cultivators the opportunity to control the climate (temperature, humidity, and light) to enhance plant production. Our present work focused on ways to control the inside temperature and inside humidity in a greenhouse. These two variables are strongly influenced by outside temperature, outside humidity, wind speed, and solar radiation.

A greenhouse climate is a nonlinear time variant multipleinput multiple-output system (MIMO) [43]. Therefore, there are many challenges in modeling and controlling a greenhouse climate. Fuzzy control is an important component of intelligent control that does not require an accurate description of the controlled system. Consequently, it does not depend on an accurate mathematical model. It has good robustness and adaptability, and it is readily accepted by the operator [44, 45]. Thus, it is very appropriate for controlling a complex system, such as a greenhouse.

L. Zadeh introduced fuzzy sets and fuzzy logic in 1965 [46] as a way of representing and manipulating data that were not precise [46]. Fuzzy logic is an extension of classical logic that allows the modeling of imperfect information and approaches 
to control problems; it mimics how a person would make decisions but in a much faster way [47]. It may be implemented in hardware or software, or both.

The fuzzifier converts the input crisp variables using the appropriate membership functions of the fuzzy linguistic variables, which are words or phrases in a natural or artificial language, not numbers [24]. This principle is illustrated in Figure 4. Figure 4 shows the proposed Fuzzy logic model for the greenhouse. The input signals are external temperature and internal temperature, external and internal humidity, wind speed, and global radiation. The output signals are the heating and extractor controllers.

In the standard fuzzy partition, each fuzzy set corresponds to a linguistic concept. For this application, low, medium, and high are the linguistic variables. For this case, we chose an Assilan-Mamdani type fuzzy logic controller. An example of a membership function set for the input is shown in Figure 5. Also, Figure 6 presents an example of fusing by Arduino language.

Furthermore, because we have six entries with three degrees of membership, 729 is the maximum number of possibilities for all the rules, according to these entries. This number was concatenated to 77 rules. Figure 7 shows an example of some rules programmed with Arduino language.

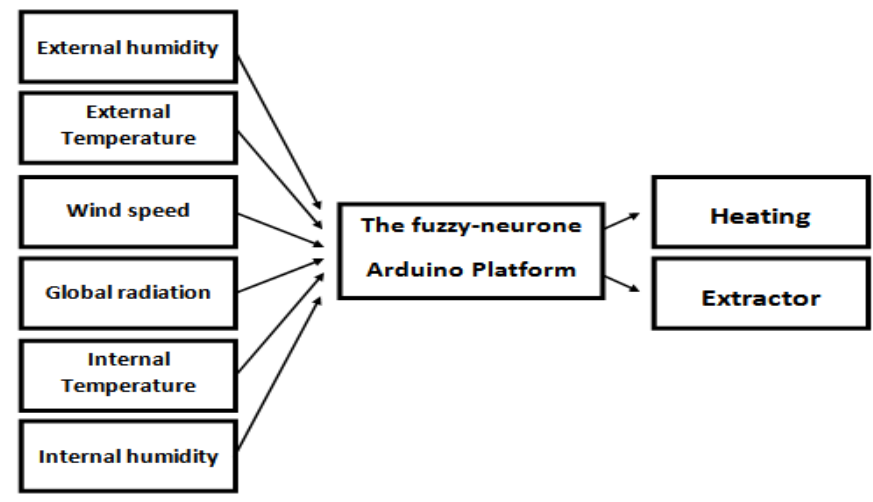

Figure 4. The proposed fuzzy logic model for the greenhouse

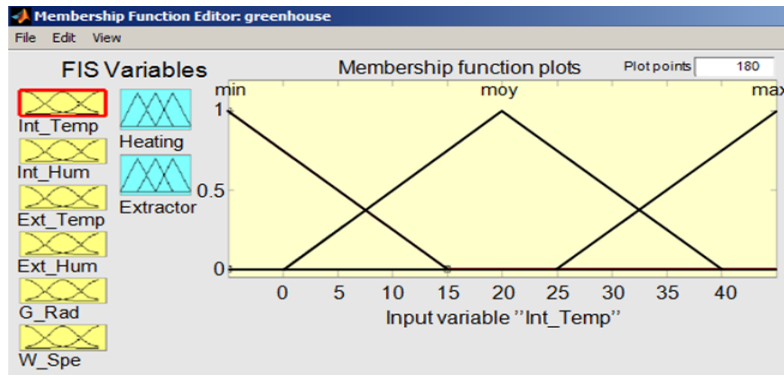

Figure 5. Membership function of external temperature

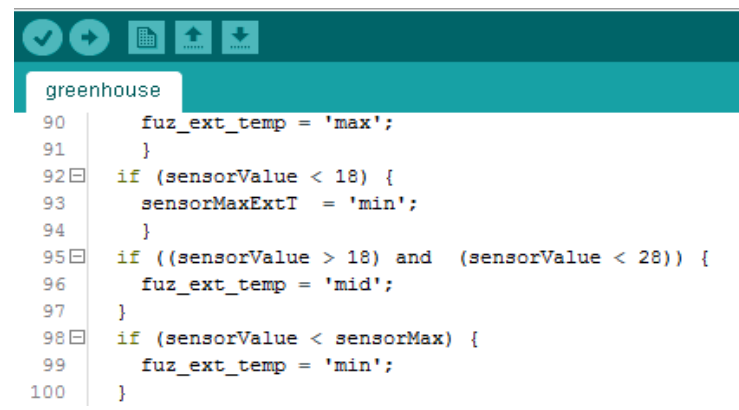

Figure 6. Example of fusing by Arduino language

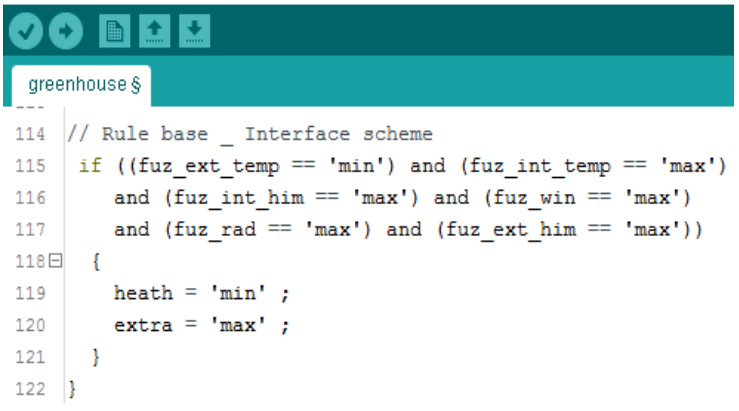

Figure 7. Example of some rules programmed with Arduino language

The fuzzy rules are written as follows:

If $x_{1}$ is $F_{1}$ and $x_{2}$ is $F_{2} \ldots$ and $x_{n}$ is $F_{n}$, then $y$ is $F_{y}$

An example of some rules as indicated below:

IF W-speed IS low AND G-radiation IS low AND H_ext IS Average AND H_int IS Average AND T_ext IS low AND T_int IS Average THEN Heating is low AND Extractor is Average

IF W-speed IS Average AND G-radiation IS Average AND H_ext IS low AND H_int IS low AND T_ext IS Average AND T_int IS Average THEN Heating is Average AND Extractor is Average

IF W-speed IS High AND G-radiation IS High AND H_ext IS High AND H_int IS Average AND T_ext IS Average AND T_int IS Average THEN Heating is Average AND Extractor is Average

IF W-speed IS Average AND G-radiation IS Average AND H_ext IS low AND H_int IS Average AND T_ext IS High AND T_int IS Average THEN Heating is Average AND is Extractor Average

IF W-speed IS low AND G-radiation IS low AND H_ext IS low AND H_int IS low AND T_ext IS High AND T_int IS High THEN Heating is low AND Extractor is Average

IF W-speed IS High AND G-radiation IS Average AND H_ext IS low AND H_int IS low AND T_ext IS High AND $\mathrm{T}$ int IS High THEN Heating is Average AND Extractor is low

In fuzzy logic, the toolbox window of the controller structure is presented in Figure 8 .

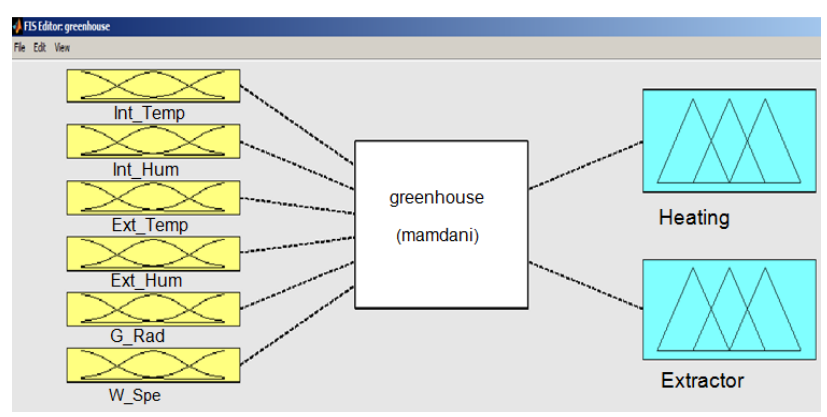

Figure 8. The toolbox window of the controller structure in fuzzy logic

\subsection{Processing part}

Real-time embedded systems are typically small in scale. Some projects require determinism hardware where logic is achieved on an ASIC or FPGA [48], but our solution is implemented in a software program on calculators. The 
Arduino UNO board was used as the base station controller [49] since it is compact, inexpensive, and has low power consumption. It is also able to process the fuzzy logic controller and eliminates the need for a computer.

The system collects data from a WSN using an Xbee shield and is configured as the coordinator, which makes it a WSN node. Then, the data detected by the sensor nodes can be locally processed with a predefined fuzzy controller and generates outputs for different inputs. The fuzzy output controller is fed to the Arduino board for the generation of a command signal corresponding to the changing input signal. Also, the commands are sent through the coordinator Xbee shield to the different actuators. At the same time, it can communicate with the internet via an ESP8266 Wi-Fi Module. The latter is a self-contained SOC with an incorporated TCP/IP protocol stack that permits any microcontroller to access a wireless network. Moreover, the data and orders are transferred to the hosting server using wireless networks. When data is received by the server, they are stored and analyzed on cloud servers. Therefore, when cultivator, engineer or users want to consult and observe data. They will use a web explorer through a computer, tablet, and smartphone, etc.

\begin{tabular}{|c|c|c|}
\hline phрМИAdmin & — 다요 Server & $12700.1 \geqslant 0$ Databa \\
\hline 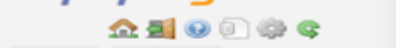 & Browse & In structure \\
\hline Recent Favorites & $\leftarrow T \rightarrow$ & $\nabla$ is \\
\hline$\Theta \infty$ & $\square$ Edit & 할 Copy $\odot$ Delete \\
\hline 50 New & Edit & ్ㅏ- Copy $\odot$ Delete \\
\hline T & $\square$ Edit & 할: Copy $\odot$ Delete \\
\hline+2 temp & Edit & ్ㅜㄹ Copy $\odot$ Delete \\
\hline Ho hum & Edit & 훌ं Copy $\odot$ Delete \\
\hline+-0 mysql & $\square \curvearrowright$ Edit & ్ㅏㅁ Copy @ Delete \\
\hline +1) performance_schema & $\square$ Edit & 함: Copy $\odot$ Delete \\
\hline $\begin{array}{l}+10 \text { phpmyadmin } \\
+-10 \text { reda }\end{array}$ & Edit & ్ㅜㄱ-ं Copy $\Theta$ Delete \\
\hline+-1 test & Edit & 하주 Copy $\odot$ Delete \\
\hline & $\square \approx$ Edit & 각- Copy $\Theta$ Delete \\
\hline & Edit & ్ㅜㄹ: Copy $\odot$ Delete \\
\hline & Edit & ₹్j-c Copy $\odot$ Delete \\
\hline & $\square$ Edit & ్ㅜㄹ Copy $\odot$ Delete \\
\hline & Edit & 학: Copy $\odot$ Delete \\
\hline
\end{tabular}

Figure 9. "arduino" database

\subsection{Server application part}

When a cultivator, an engineer, or other users want to consult data that was sent from the Arduino part, they need to type the website address or domain into their browser through a set of devices (laptops, smartphones, PCs). The selected device will then connect to the server, and web pages will be delivered to the users through the browser. The Cloud server will receive, save, compute, and analyze data. For all of these functions, the users in that area have chosen the use of the cross-platform web server XAMPP set, the house Apache server that comes bundled with MySQL database, PHP (a server-side scripting language), Perl (another server-side scripting language), the FileZilla FTP Server, and the Mercury Mail Transport System.

A database in MySQL, named "arduino", can be implemented as shown in Figure 9.

\section{RESULTS DISCUSSION}

The system structure is comprised of several elements as shown in Figure 3. Firstly, we completed a series of tests to detect possible problems in the implementation, with the intention of ascertaining that is performed correctly. Secondly, all parts were assembled, and an internet $\mathrm{Wi}-\mathrm{Fi}$ connection was established. The access to the web page from a smartphone, tablet, or laptop allows the user to display received data in a digital or graph form.

The designed system collects global data which are external temperature, external humidity levels, wind speed, global radiation, and finally actuator extractor. Indeed, we also collect internal data from two different greenhouses which are temperature and humidity using wireless sensors every 30 minutes. The results have shown the differences between the greenhouse parameters without controller (Figure 10) and the greenhouse parameters with controller (Figure 11). Figure 10 illustrated that the confined air reaches an average temperature of $41.80^{\circ} \mathrm{C}$ when the average air of outside temperature is $26.00^{\circ} \mathrm{C}$. On the other hand, relative humidity in the interior is lower at $19.1 \%$. This remarkable humidity diminution makes the greenhouse converts the external environment from dry warm to a desert type micro-climate.

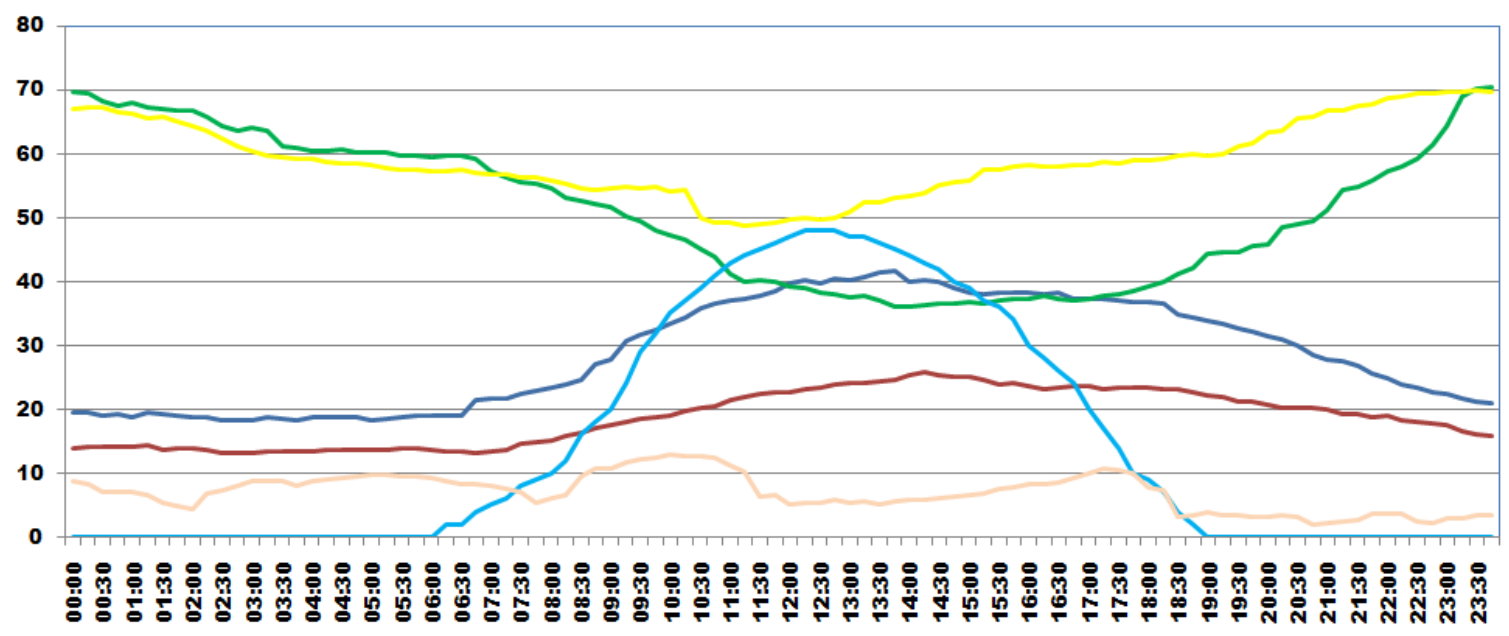

—inside temperature —outside temperature —inside humidity $\quad$ outside humidity _ $\quad$ global radiation $\quad$ wind speed

Figure 10. Internal and external greenhouse parameters without controller 


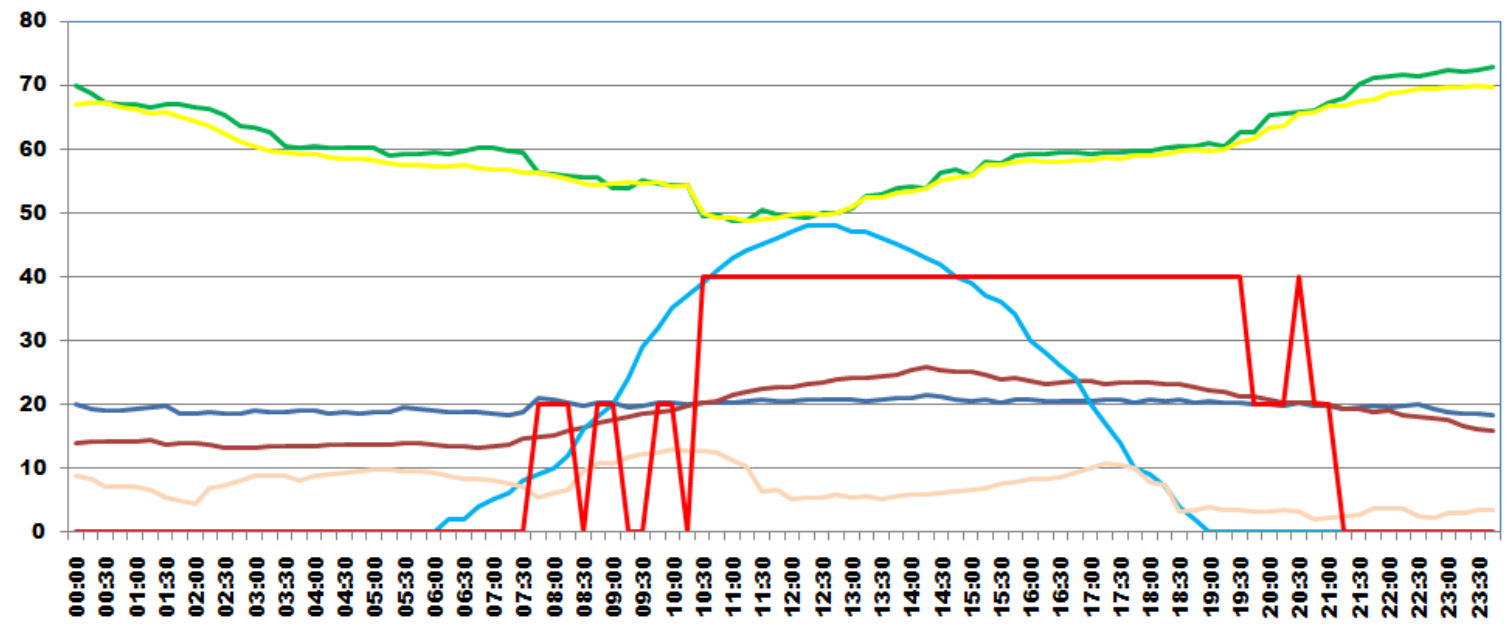

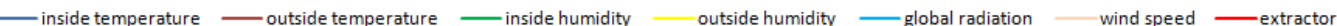

Figure 11. Internal and external greenhouse parameters with controller

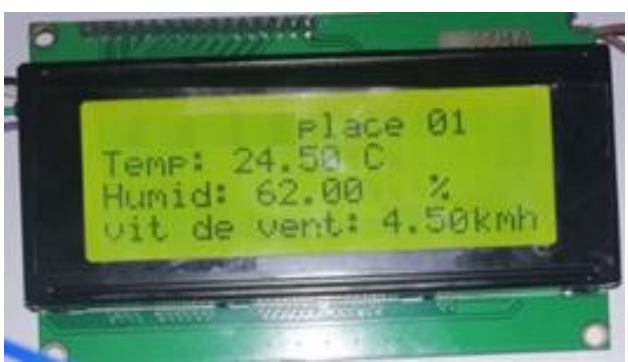

Figure 12. An example of the parameters displayed by LCD interface with Arduino

On the other hand, as shown in Figure 11, it can be observed that the internal temperature reaches an average temperature of $20{ }^{\circ} \mathrm{C}$; this is due to the performance of the fuzzy logic controller. It is worth remarking here that when the inside temperature will start to rise with outside temperature, the operator extractor intervenes to moderate the atmosphere with low, and when the outside temperature more increased, he works with height. Also, there is significant stability of inside humidity near outside humidity. As a result, that will create an optimal environment for plants in the greenhouse

Figure 12 shows the parameters over the LCD Arduino interface for Greenhouse Monitoring System.

It can be asserted that the greenhouse cycle is seasonal, Thus, there are no significant climate changes. Also, the growth stages of any plant are not immediate. Therefore, the changes in its climatic requirements are divergent. So, it can be controlled manually by changing and controlling the input signals, at the beginning of each growth stage by programing the Arduino.

Also, to have access to information, we developed interfaces, making the dialogue between humans and machines easy. In addition, they have to take into account the set of ergonomic choices, such as readability, comprehensibility, and easy-to-understand features, for the whole range of potential users.

Figure 13 shows how the data are received by the server. The data arrive sequentially in the digital form on a single block containing internal temperature and humidity values with the time and date of the received data.

Also, data can be accessed from web pages with any internet connected device. This device allows the user to display and interact with the server data in real-time.

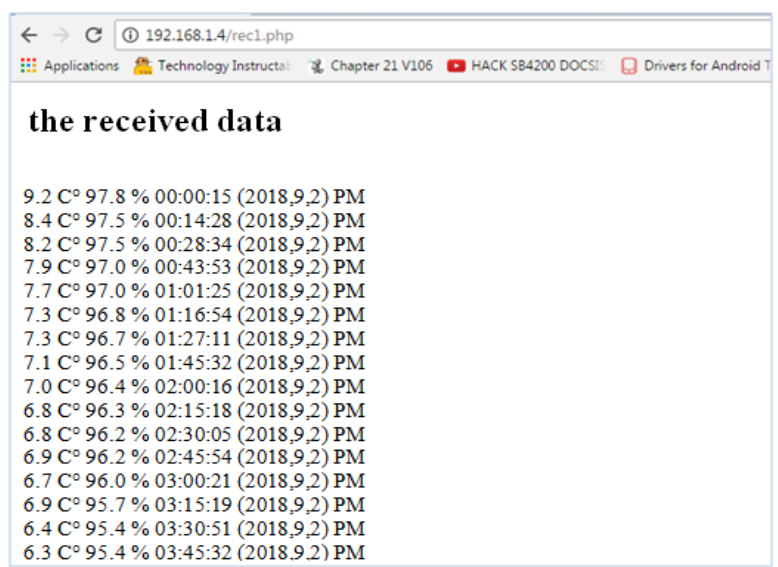

Figure 13. Received internal temperature and humidity data in digital form

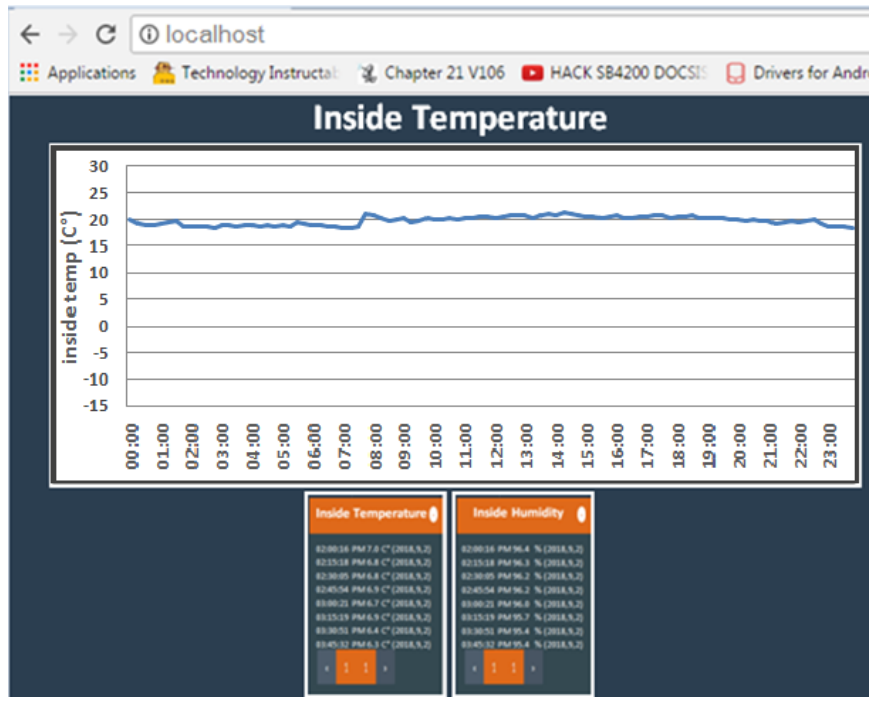

Figure 14. Received data (Inside Temperature) in graph form

As shown in Figures 14 and 15, the web page consists of two sections; one to display parameters in numerical form. This allows the user to monitor the greenhouse climate. The data includes the internal temperature [in degrees Celsius $\left({ }^{\circ} \mathrm{C}\right)$ ], time, date, and the internal humidity [(in percent)], time, date. It can be noticed that the visualization of data in graph form is 
a powerful tool [50] to analyze and monitor the greenhouse climate.

Also, the web page displays numerical data values received in graph form (see also Figures 14 and 15), which show the graph of the variation of internal temperature and the internal humidity according to time, respectively. The two types of data visualization allow for genuine analyses and accurate discussions.

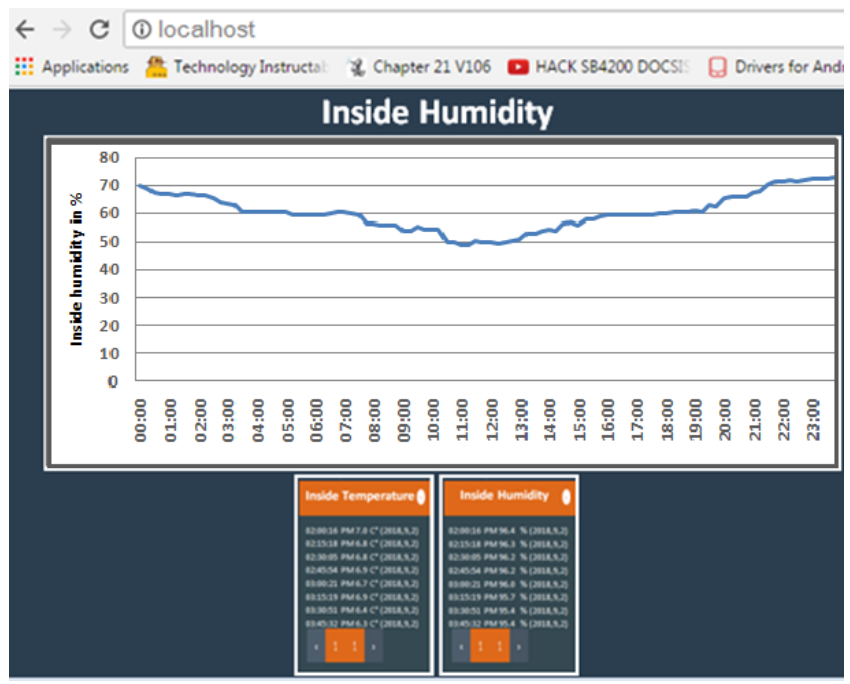

Figure 15. Received data (Inside humidity) in graph form

\section{CONCLUSION}

The system can monitor and intelligently control the greenhouse climate through the internet based on IoT technology. One major advantage of the design is the integration of the WSN node and the fuzzy control program using a simple Arduino Uno board. Also, an internet connection unit allows the system to be tracked in real-time. Hence, this creates an ideal climate for the proper growth of plants with effective telemonitoring, opportunities for data analysis and more accurate discussion.

In our future work, we can add a remote change of input signals controller, and we plan to extend the system to include irrigation, the regulation of $\mathrm{CO}_{2}$ level, etc., as well to provide more precise control for greenhouse farming. To improve the operational safety of the system, we can integrate redundant processing in the server. Verification might be accomplished by comparing results. If the results agree, the system is operating properly; if not, alerts messages can be transmitted. Such design is of great importance and could be applied on a wider basis in many monitoring, command, and data collection applications.

\section{ACKNOWLEDGMENT}

This work was supported by the Directorate General for Scientific Research and Technological Development (DGRSDT) of Algeria.

\section{REFERENCES}

[1] Baulcombe, D., Crute, I., Davies, B., Dunwell, J., Gale,
M., Jones, J., Pretty, J., Sutherland, W., Toulmin, C. (2009). Reaping the benefits: Science and the sustainable intensification of global agriculture. The Royal Society. London, 72. ISBN 9780854037841

[2] Bollani, L., Bonadonna, A., Peira, G. (2019). The Millennials' concept of sustainability in the food sector. Sustainability, 11(10): 2984. https://doi.org/10.3390/su11102984

[3] Somov, A., Shadrin, D., Fastovets, I., Nikitin, A., Matveev, S., seledets, I., Hrinchuk, O. (2018). Pervasive agriculture: IoT-enabled greenhouse for plant growth control. IEEE Pervasive Computing, 17(4): 65-75. http://doi.org/10.1109/mprv.2018.2873849

[4] Benke, K., Tomkins, B. (2017). Future food-production systems: Vertical farming and controlled-environment agriculture, Sustainability: Sci Pract Policy, 13(1): 13-26. http://doi.org/10.1080/15487733.2017.1394054

[5] Chen, F., Qin, L., Li, X., Wu, G., Shi, C. (2017). Design and implementation of ZigBee wireless sensor and control network system in greenhouse. 36th Chinese Control Conference (CCC), China, pp. 8982-8986. http://doi.org/10.23919/ChiCC.2017.8028786

[6] The Future of Food and Agriculture Trends and Challenges. (2017). Food and agriculture organization of the united nations, Rome. http://www.fao.org

[7] Dieisson, P., Paulo, D.W., Edson, T., Caroline, F., Giana, V.M. (2018). Scientific development of smart farming technologies and their application in Brazil. Information Processing in Agriculture, 5(1): 21-32. https://doi.org/10.1016/j.inpa.2017.12.002

[8] Perera, C., Zaslavsky, A., Christen, P., Georgakopoulos, D. (2014). Context aware computing for the internet of things: A survey. IEEE Communications Surveys and Tutorials, 16(1): 414-454. https://doi.org/10.1109/surv.2013.042313.00197

[9] Putra, R.H., Kusuma, F.T., Damayanti, T.N., Ramadan, D.N. (2019). IoT: Smart garbage monitoring using android and real time database. TELKOMNIKA Telecommunication Computing. Electronics and Control, 17(3):

1483-1491. https://doi.org/10.12928/telkomnika.v17i3.10121

[10] Li, Y. (2018). Design and implementation of intelligent travel recommendation system based on internet of things. Ingénierie des Systèmes d'Information, 23(5): 159-173. https://doi.org/10.3166/ISI.23.5.159-173

[11] Sarma, S., Brock, D., Ashton, K. (1999). The networked physical world: Proposals for the next generation of computing commerce, and automatic identification. AutoID Center White Paper.

[12] Wolfert, S., Ge, L., Verdouw, C., Bogaardt, M.J. (2017). Big data in smart farming a review. Agricultural Systems, 15(3):

69-80. https://doi.org/10.1016/j.agsy.2017.01.0232017

[13] Perera, C., Jayaraman, P., Zaslavsky, A., Christen, P., Georgakopoulos, D. (2013). Dynamic configuration of sensors using mobile sensor hub in internet of things paradigm. IEEE 8th International Conference on Intelligent Sensors, Sensor Networks and Information Processing (ISSNIP), Melbourne, Australia, pp. 473-478 https://doi.org/10.1109/issnip.2013.6529836

[14] Haider, M.J., Rosdiadee, N., Sadik, K.G., Aqeel, M.J., Mahamod, I. (2017). Energy-efficient wireless sensor networks for precision agriculture: A review. Sensors, 17(8): 1-45. https://doi.org/10.3390/s17081781 
[15] Almeida, F. (2018). Big data: Concept, potentialities and vulnerabilities. Emerging Science Journal, 2(1): 1-10. http://dx.doi.org/10.28991/esj-2018-01123

[16] Li, X., Wang, M., Wang, H., Yu, Y., Qian, C. (2019). Toward secure and efficient communication for the internet of things. IEEE/ACM Transactions on Networking, 17(2): 621-634. https://doi.org/10.1109/tnet.2019.2893249

[17] Cai, H., Xu, B., Jiang, L., Vasilakos, A.V. (2016). IoTbased big data storage systems in cloud computing: perspectives and challenges. IEEE Internet of Things Journal, $4(1)$ :

$75-87$. https://doi.org/10.1109/jiot.2016.2619369

[18] Khan, R., Khan, S.U., Zaheer, R., Khan, S. (2012). Future internet: The internet of things architecture, possible applications and key challenges. In 2012 10th International Conference on Frontiers of Information Technology, pp. 257-260. https://doi.org/10.1109/fit.2012.53

[19] Mavrogiorgou, A., Kiourtis, A., Touloupou, M., Kapassa, E., Kyriazis, D. (2019). Internet of medical things (IoMT): Acquiring and transforming data into HL7 FHIR through 5G network slicing. Emerg. Sci. J., 3(2): 64-77. https://doi.org/10.28991/esj-2019-01170

[20] Mars, D., Gammar, S. M., Lahmadi, A., Saidane, L.A. (2019). Using information centric networking in internet of things: A survey. Wireless Personal Communications, 105(1): 87-103. https://doi.org/10.1007/s11277-0186104-8

[21] Chen, W.H., Ballance, D.J., Gawthrop, P.J. (2003). Optimal control of nonlinear systems: A predictive control approach. Automatica, 39(4): 633-641. https://doi.org/10.1016/s0005-1098(02)00272-8

[22] Li, C., Chiang, T.W., Yeh, L.C. (2013). A novel selforganizing complex neuro-fuzzy approach to the problem of time series forecasting. Neurocomputing, 99: 467-476. https://doi.org/10.1016/j.neucom.2012.07.014

[23] Shamshiri, R., Fatemeh, K., Ting, K.C., Kelly, R.T., Ibrahim, A.H., Cornelia, W., Desa, A., Zahra, M.S. (2018). Advances in greenhouse automation and controlled environment agriculture: A transition to plant factories and urban farming. International Journal of Agricultural and Biological Engineering, 11(1): 1-22. https://doi.org/10.25165/j.ijabe.20181101.3210

[24] Kapitanova, K., Son, S.H., Kang, K.D. (2012). Using fuzzy logic for robust event detection in wireless sensor networks. Ad Hoc Networks, 10(4): 709-722. https://doi.org/10.1016/j.adhoc.2011.06.008

[25] Argo, B.D., Hendrawan, Y., Ubaidillah, U. (2019). A fuzzy micro-climate controller for small indoor aeroponics systems. TELKOMNIKA, 17(6): 3019-3026. https://doi.org/10.12928/telkomnika.v17i6.12214

[26] Mulenga, D., Siziya, S. (2019). Indoor air pollution related respiratory ill health, a sequel of biomass use. SciMedicine Journal, 1(1): 30-37. http://dx.doi.org/10.28991/SciMedJ-2019-0101-5

[27] Mirzaee-Ghaleh, E., Omid, M., Keyhani, A., Dalvand, M.J. (2015). Comparison of fuzzy and on/off controllers for winter season indoor climate management in a model poultry house. Computers and Electronics in Agriculture, 110: 187-195. https://doi.org/10.1016/j.compag.2014.11.017

[28] Manonmani, A., Thyagarajan, T., Sutha, S. (2017). ANN based modeling and control of GHS for winter climate.
In 2017 Trends in Industrial Measurement and Automation (TIMA), $\quad$ pp. 1-7. https://doi.org/10.1109/TIMA.2017.8064816

[29] Azaza, M., Tanougast, C., Fabrizio, E., Mami, A. (2016). Smart greenhouse fuzzy logic based control system enhanced with wireless data monitoring. ISA Transactions, 61: 297-307. https://doi.org/10.1016/j.isatra.2015.12.006

[30] Pahuja, R., Verma, H.K., Uddin, M. (2011). Design and implementation of fuzzy temperature control for WSN applications. Int. J. Comput. Sci. Netw. Secur, 11(2): 110.

[31] Karunakar, P., Anusha, C.A. (2014). Comparative study of wireless protocols: Bluetooth, UWB, ZigBee, and WiFi. Advance in Electronic and Electric Engineering, 4(6): 655-662.

[32] Lee, J.S., Su, Y.W., Shen, C.C. (2007). A comparative study of wireless protocols: Bluetooth, UWB, ZigBee, and Wi-Fi. In IECON 2007-33rd Annual Conference of the IEEE Industrial Electronics Society, pp. 46-51. https://doi.org/10.1109/iecon.2007.4460126

[33] Guo, W., Healy, W.M., Zhou, M. (2011). Interference impacts on ZigBee-based wireless mesh networks for building automation and control. In 2011 IEEE International Conference on Systems, Man, and Cybernetics, pp. 3452-3457. https://doi.org/10.1109/icsmc.2011.6084203

[34] Sagheer, A., Mohammed, M., Riad, K., Alhajhoj, M. (2021). A cloud-based IoT platform for precision control of soilless greenhouse cultivation. Sensors, 21(1): 1-28. https://doi.org/10.3390/s21010223

[35] Rekibi, F. (2015). Analyse compétitive de la filière tomate sous serre. Cas de la Wilaya de Biskra. M.S. thesis. Biskra: Mohamed Kheider University.

[36] DSA. (2012). Direction Des Services Agricoles. Biskra province.

[37] Damagnez, J., van der Borg, H.H. (1981). Optimisation de la croissance des plantes sous serre par le controle du microclimate. Commission of the European Communities, Coordination of Agricultural Research, ISHS, Workinggroup GCC, ISHS, and Commission Horticultural Engineering: Sasanquas, Vaucluse, France. Acta horticulturae, ISHS: 1-4.

[38] El-Gayar, S., Negm, A., Abdrabbo, M. (2018). Greenhouse operation and management in Egypt. In Conventional Water Resources and Agriculture in Egypt, pp. 489-560. https://doi.org/10.1007/698_2017_230

[39] Ojha, T., Misra, S., Raghuwanshi, N.S. (2015). Wireless sensor networks for agriculture: The state-of-the-art in practice and future challenges. Computers and Electronics in Agriculture, 118: 66-84. https://doi.org/10.1016/j.compag.2015.08.011

[40] Sidhu, B., Singh, H., Chhabra, A. (2007). Emerging wireless standards-wifi, zigbee and wimax. World Academy of Science, Engineering and Technology, 25 308-313. https:// doi.org/10.5281/zenodo.1077149

[41] Chakkor, S., Cheikh, E.A., Baghouri, M., Hajraoui, A. (2014). Comparative performance analysis of wireless communication protocols for intelligent sensors and their applications. arXiv preprint arXiv:1409.6884. https://doi.org/10.14569/ijacsa.2014.050413

[42] Zhou, Y., Guo, X., Zhou, M., Wang, L. (2007). A design of greenhouse monitoring \& control system based on ZigBee wireless sensor network. In 2007 International 
Conference on Wireless Communications, Networking and Mobile Computing, pp. 2563-2567. https://doi.org/10.1109/wicom.2007.638

[43] Yousefi, M.R., Hasanzadeh, S., Mirinejad, H., Ghasemian, M. (2010). A hybrid neuro-fuzzy approach for greenhouse climate modeling. In 2010 5th IEEE International Conference Intelligent Systems, pp. 212217. https://doi.org/10.1109/is.2010.5548375

[44] Shi, X.M., Hao, Z.Q. (2008). Fuzzy Control and MATLAB Simulation. Beijing. China: Tsinghua University Press \& Beijing Jiaotong Univertity Press.

[45] Dou, L.L., Xu, W.Z., Li, X., Li, Q. (2007). Application of fuzzy control in greenhouse environment control systems. Journal of China Jiliang University, 18(1): 3437.

[46] Zadeh, L.A. (1996). Fuzzy sets. In Fuzzy sets, fuzzy logic, and fuzzy systems: Selected papers by Lotfi A Zadeh, pp. 394-432.

[47] Agusta, S., Girsang, A.S. (2018). Effort estimation development model for web-based mobile application using fuzzy logic. Telkomnika, 16(5): 2082-2090. https://doi.org/10.12928/telkomnika.v16i5.6561
[48] Jahanirad, H., Karam, H. (2017). BIST-based Testing and Diagnosis of LUTs in SRAM-based FPGAs. Emerging Science Journal, 1(4): 216-225. https://doi.org/10.28991/ijse-01125

[49] Gibb, M. (2010.). New media art, design, and the Arduino microcontroller: A malleable tool. PhD thesis, Pratt Institute.

[50] Nowghabi, A., Talebzadeh, A. (2019). Psychological influence of advertising billboards on city sight. Civil Engineering Journal, 5(2): 390-397. https://doi.org/10.28991/cej-2019-03091253

\section{NOMENCLATURE}

$\begin{array}{ll}\text { W-speed } & \text { Wind-speed } \\ \text { G-radiation } & \text { Global-radiation } \\ \text { H_ext } & \text { external humidity } \\ \text { H_int } & \text { Internal humidity } \\ \text { T_ext } & \text { external temperature } \\ \text { T_int } & \text { Internal temperature }\end{array}$

